during the contrast injection. In the fronto-temporal area, diffuse cerebral telagiectasia were seen. At one week after the first attempt of angiography, serial angiography was carried out to make a circulatory state clear. No sooner had the ' $x$-rays been taken than the patient fell into coma. In spite of an intensive treatment, the patient expired 48 hours after the rupture.

Autopsy displayed marked edema on both hemispheres, and a large hematoma in the left fronto-temporal lobe. An intracavernous aneurysm was found to be isituated at the dorsolateral aspect of the left internal carotid artery. Microscopic Eexamination showed that the left internal carotid artery, at the level of immediately "proximal to the aneurysm, was characterized by partial separation of the media being edematous, intimal thickening in part, proliferation of much waved internal 3astica. At A-1 of the left anterior cerebral artery, the intima was partly thick .nd fibrotic; the internal elastica was much waved and dupricated; the media and idventitia markedly edematous and fibrotic. In the left Sylvian fissure, the "nternal elastica of the middle cerebral artery was separated; the intima, media, lind adventitia were in the same changes as A-1 of the anterior cerebral artery. 'ial vessels were found to be increased in the number and dilated, with the media nd adventitia thinner. Capillaries were also seen dilated. Extravasation, by nd large, was found in pial arterioles, penetrating arteries, and capillaries; not bserved in the venous system.

liscussion to $\mathbf{f - 3}$.

\title{
The Case of Carotid Cavernous Fistula repeated several fetal Subarachnoid Bleeding due to the Rupture of Three Intracranial Aneurysms
}

\author{
Yuji Miyazaki and Katsumi Suematsu \\ Department of Neurosurgery, Sapporo Medical College
}

A case who had three intracranial aneurysms with carotid cavernous fistula $d$ had died after several subarachnoid bleeding due to rupture of aneurysms $s$ reported.

The patient, a man of 32 , had injuried his head, had been a continuous roar the left side of his head 2 months later after head injury. He had noted irked protusion of his left eye 3 months later, under diagnosis of a carotid 'ernous fistura, the first operation (ligation of left common carotid artery) was te in 1952 and second operation (ligation of left internal and external artery) $s$ done in 1965 . In 1968 , he died after several subarachnoid bleeding.

Three aneurysms were discovered by autopsy-one at the bifrication of left erior cerebral artery and left middle cerebral artery, the other in the left 
cerebellar artery. Each aneurysm had a large intracerebral hematoma in the frontal lobe and in the left cerebellar hemisphere. This reported case lead the conclusion that aneurysm of carotid artery in the cavernous sinus was one of a congenital origin, because there were three aneurysms simultaneously and atheromatous change was observed in the wall of aneurysms.

\title{
f-4. Paraparesis associated with Cerebrovascular Lesion
}

\author{
Syun Yamasaki, Koreaki Mori, Junkoh Yamashita, Yasuaki Yoshida \\ and Taro Fukumitsu \\ Department of Neurosurgery, Shizuoka Rosai Hospital
}

Paraplegia or paraparesis is usually considered to be caused by lesions of the spinal cord. However, arteriosclerotic or senile paraplegia results more frequently from cerebrovascular lesion. We reported two cases of cerebral paraplegia of sudden onset which occurred in older age.

Case 1. A 54-year-old male who had suffered from hypertension for several years had a sudden attack of paresis and numbness in the lower extremities four years prior to admission to our clinic. Gradually he regained his muscular power and became ambulant with a stick. Examination revealed spastic paresis and hypesthesia of the lower extremities and hyperactive patellar jerk. Electroencephalography showed slow $\alpha$ rhythm but no marked asymmetry. Right carotid angiography showed occlusion of the proximal the right middle cerebral and the right pericallosal artery. Pneumoencephalography revealed moderate right hemiatrophy.

Case 2. A 68-year-old male with situs inversus viscerum had suddenly developed paraparesis and tingling in the lower extremities and urinary incontinence one year and a half before admission. Although paraparesis had improved gradually, insomnia, paresthesia of the lower extremities and chest discomfort remained. On examination, paraparesis and paresthesia of the lower extremities, presbyacusis and arteriosclerotic optic fundi were observed. Electroencephalography revealed symmetrical slow $\alpha$ rhythm with low voltage $\theta$ waves. A saccular aneurysm of the anterior communicating artery with atheromatous changes in the internal carotid and anterior cerebral arteries was discovered by right carotid angiography.

From the functional anatomical point of view, cerebral paraparesis or paraplegia is considered to occur in lesions of the parasagittal region due to a meningioma arising from the falx, thrombosis of the superior sagittal sinus and of the un-paired anterior cerebral arteries, etc.

Clinically it is important to distinguish cerebral paraplegia from spinal one. Especially paraplegia associated with cerebrovascular lesion should be differen ciated from anterior spinal artery syndrome. The key point of differentiation 